\title{
The telomere protein tankyrase 1 regulates DNA damage responses at telomeres
}

\author{
Sandy Chang \\ Department of Laboratory Medicine, Yale University School of Medicine, New Haven, CT 06520, USA
}

Commentary on: Ryan C. Dregalla, Junqing Zhou, Rupa R. Idate, Christine L.R. Battaglia, Howard L. Liber, and Susan M. Bailey. Regulatory roles of tankyrase 1 at telomeres and in DNA repair: suppression of T-SCE and stabilization of DNA-PKCS Aging. 2010; 2: this issue

E-mail: s.chang@yale.edu

The proliferative potential of eukaryotic cells is critically dependent upon the maintenance of functional telomeres, the protein-DNA complexes that cap the ends of chromosomes. A paper published in this issue of Aging describes that the telomere protein tankyrase 1 regulates DNA damage responses at telomeres.

Telomeres are composed of TTAGGG repeats that associate with a six-protein telomere-specific complex termed shelterin, composed of telomeric-repeat binding factor 1 (TRF1), TRF2, TRF1 interacting protein 2 (TIN2), protection of telomeres 1 (POT1), the POT1 and TIN2 interacting protein TPP1 and the transcriptional repressor/activator protein RAP1 [1]. The shelterin complex comprises the physical termini of chromosomes and serves to prevent chromosomal ends from being recognized as DNA double-strand breaks (DSBs). The synthesis and maintenance of telomeric repeats are mediated by telomerase, a specialized ribonucleoprotein complex [2]. In the absence of telomerase, the failure of DNA polymerase to fully synthesize terminal ends of the lagging DNA strand leads to progressive telomere shortening with each round of replication. In human somatic tissues, the strict down-regulation of telomerase accounts for the age-dependent decline in telomere lengths in somatic cells. Studies have documented a decrease in telomere length in several human epithelial cell types, ranging from 50-100 bp per population doubling, for a total lifetime loss of approximately $2-4 \mathrm{~kb}$ [3]. This rate of telomere length attrition would be significant in longlived organisms such as humans.

A large body of work in human cell culture systems and mouse models has documented the biological and genomic consequences of telomere attrition and how these consequences relate to the development of premature aging and cancer [4]. Primary human cells have a limited replicative potential due to insufficient telomerase, resulting in the progressive shortening of telomeres with each cell division, eventually leading to the onset of replicative senescence. Replicative senescence has been shown to be due to critically shortened (dysfunctional) telomeres activating the p53dependent DNA damage response checkpoint. Rare cells that stochastically lose p53 or Rb function bypass this senescence checkpoint and progress towards cancer. These cells continue to shorten their telomeres, resulting in entry into a phase of rampant chromosomal instability termed crisis, characterized by end-to-end chromosomal fusions. Depending on how fused chromosomes are resolved, loss of heterozygocity of tumor suppressors and/or amplification of oncogenes could lead to a pro-cancer genotype. Telomerase is reactivated in the majority of human carcinomas [5], supporting the hypothesis that telomerase reactivation is critically important for initiated cancer lesions to progress to frank malignancies, since it removes the short-telomere barriers that are inhibitory to tumor progression.

A subset of human cancers utilizes a telomeraseindependent, alternative lengthening of telomeres (ALT) mechanisms to maintain telomere length [6]. Although the exact molecular mechanisms underlying ALT in mammalian tumors remain unclear, it is likely that ALT depends upon activation of the homologous recombination (HR) repair pathway. The elegant cytogenetic technique Chromosome-Orientation (CO)FISH can be used to visualize HR within telomeric sequences [7,8]; stand-specific telomere probes are utilized to determine whether $\mathrm{HR}$ has taken place between telomeres of sister chromatids at/after DNA 
replication. This exchange, termed telomere-sister chromatid exchange (T-SCE) is dramatically elevated in ALT cells [9-11], suggesting that hyper telomeric recombination may be a hallmark of this cell type. In addition, disrupting components of the shelterin complex, including TPP1/POT1 and TRF2 (in combination with DNA repair factors involved in the classical non-homologous end joining [C-NHEJ], Ku70 and 53BP1) also results in elevated T-SCE [12-14]. These results suggest that aberrant telomere-telomere HR is actively repressed by components of the shelterin complex.

In the current issue of AGING, Dregalla and colleagues extend upon these observations to reveal that removal of tankyrase 1, a telomere-associated poly(adenosine diphosphate [ADP]-ribose) polymerase (PARP), also results in increased frequencies of T-SCE [15]. Tankyrase 1 plays a critical role in regulating the quantity of TRF1 at telomeres, since tankyrase 1 mediated poly(ADP-ribosyl)ation of TRF1 liberates it from telomeres [16,17]. Surprisingly, Dregalla et al. found that depletion of Tankyrase 1 also resulted in the rapid, proteosome-mediated degradation of DNA-PKcs, another major component of the NHEJ pathway. Other proteins involved in NHEJ, including Ku86 and ATM, were unaffected upon tankyrase 1 depletion, suggesting that DNA-PKcs stability specifically depends on tankyrase 1. Administering the small molecule PARP inhibitor XAV939 to cells also resulted in rapid depletion of DNA-PKcs, indicating specificity of the tankyrase 1 PARP domain for modulating DNA-PKcs stability. It is important to note that removal of DNAPKcs by itself did not result in increased T-SCE, suggesting tankyrase 1 suppresses T-SCE independent of its role in mediating DNA-PKes stability.

Why does aberrant recombination have to be repressed at telomeres? Recent data suggest that telomeres employ different shelterin components to prevent uncapped telomeres from engaging in distinct DNA damage signaling pathways. For example, TRF2 specifically represses ATM signaling [18,19], and removal of TRF2 elicits C-NHEJ at telomeres that requires ATM, the Mre11-Rad50-NBS1 (MRN) complex and 53BP [20-23]. In contrast, TPP1-POT1 specifically represses the ATR pathway [18-20]. Coupled with these observations, recent observations suggest that distinct DNA repair pathways at telomeres are also repressed by specific shelterin components. Removal of TRF2 resulted in the activation of Ligase 4dependent C-NHEJ-mediated end-to-end chromosome fusions. In contrast, removal of TPP1-POT1 from telomeres resulted in increased T-SCEs and chromosome fusions mediated by a Ligase 4 indepen- dent, alternative-NHEJ (A-NHEJ) pathway [14]. ANHEJ is a evolutionarily conserved repair pathway in which double strand break repair products display short tracks of microhomology at the repair junctions and the joining reaction utilizes DNA Ligase III [24]. Since DNA-PKcs is a component of C-NHEJ, it is likely that the elevated chromosome fusions observed by Dregalla et al. when tankyrase 1 is depleted (and DNA-PKcs levels are concomitantly reduced) is due to activation of A-NHEJ-mediated repair. In support of this notion, another recent report indicates that DNA-PKcs plays a role in repressing A-NHEJ at telomeres [25].

Elevated T-SCEs has also been shown recently to accelerate entry into replicative senescence [26]. Increased T-SCE levels are observed in human cell lines in which the WRN protein has been depleted by siRNA, and in cells isolated from a premature aging mouse model of Werner Syndrome (WS), in which both the gene encoding the WRN helicase and telomerase are deleted [11,26,27]. It was previously thought that unequal T-SCEs could confer a proliferative advantage to cells that stochastically acquired the longer telomeres, enabling escape from cellular senescence [7]. However, in silico modeling revealed that increased TSCEs favored entry of telomerase null cells into replicative senescence [26]. Compared to cells without T-SCE, critically shortened telomeres in cells with elevated T-SCE appear earlier and in greater numbers [28]. This unexpected result suggests that the elevated T-SCE frequencies observed in ALT cells may reflect one mechanism of generating the high proportion of very short telomere lengths characteristic of this cell type [29]. Unequal T-SCE is however unlikely to endow ALT cells with unlimited replicative potential. Perhaps the elevated T-SCEs observed in ALT cells is symptomatic of a general heightened propensity towards recombination, but is not the cause of telomere lengthening. Additional mechanisms, including but not limited to a template copy mechanism initiated by short dysfunctional telomeres, might be responsible for generating new telomere DNA.

The notion that elevated T-SCE promotes cellular senescence is likely to have interesting biological consequences. For example, in the setting of an intact p53 checkpoint, replicative senescence initiated by critically shortened telomeres has been shown to be as potent as apoptosis in suppressing tumorigenesis in vivo [30,31]. In these mouse models of cancer with dysfunctional telomeres, senescent markers were prominent in all tissues examined, suggesting that global activation of the senescence program potently inhibits tumor progression in vivo - thereby placing cellular senescence on an equal footing as apoptosis in 
tumor suppression. However, tumor suppression comes at a price, since signs of premature aging have been observed in some tumor-resistant mice bearing dysfunctional telomeres [30].

Finally, the study by Dregalla and colleagues provides insight into targeting tankyrase 1 in the clinic. Manipulation of tankyrase 1 protein levels and/or inhibition of tankyrase 1 PARP activity would be expected to compromise C-NHEJ mediated DNA damage repair by DNA-PKcs. In a similar fashion to the therapeutic strategy of using PARP inhibitors against BRCA1/2 mutation carriers [32], a therapeutic strategy may be devised to combine radiation therapy with tankyrase 1 inhibition in BRCA1/2 associated breast cancers [33].

\section{REFERENCES}

1. de Lange T. Shelterin: the protein complex that shapes and safeguards human telomeres. Genes Dev 2005; 19:2100-2110.

2. Greider CW. Telomeres; Telomerase and Senescence. Bioessays 1990; 12:363-369.

3. Harley CB, Kim NW, Prowse KR, Weinrich SL, Hirsch KS, West MD, Bacchetti S, Hirte HW, Counter CM, Greider CW, et al. Telomerase, cell immortality, and cancer. Cold Spring Harb Symp Quant Biol 1994; 59:307-315.

4. Deng Y, Chan SS, Chang S. Telomere dysfunction and tumour suppression: the senescence connection. Nat Rev Cancer 2008; 8:450-458.

5. Kim NW, Piatyszek MA, Prowse KR, Harley CB, West MD, Ho PLC, Coviello GM, Wright WE, Weinrich SL, Shay JW. Specific Association of Human Telomerase Activity With Immortal Cells and Cancer. Science 1994; 266:2011-2015.

6. Cesare AJ, Reddel RR. Alternative lengthening of telomeres: models, mechanisms and implications. Nat Rev Genet 2010; 11:319-330.

7. Bailey SM, Brenneman MA, Goodwin EH. Frequent recombination in telomeric DNA may extend the proliferative life of telomerase-negative cells. Nucleic Acids Res 2004; 32:3743-3751.

8. Bailey SM, Goodwin EH, Cornforth MN. Strand-specific fluorescence in situ hybridization: the CO-FISH family. Cytogenet Genome Res 2004; 107:14-17.

9. Londono-Vallejo JA, Der-Sarkissian H, Cazes L, Bacchetti S, Reddel RR. Alternative lengthening of telomeres is characterized by high rates of telomeric exchange. Cancer Res 2004; 64:23242327.

10. Bechter $\mathrm{OE}$, Zou $\mathrm{Y}$, Walker W, Wright WE, Shay JW. Telomeric recombination in mismatch repair deficient human colon cancer cells after telomerase inhibition. Cancer Res 2004; 64:3444-3451.

11. Laud PR, Multani AS, Bailey SM, Wu L, Ma J, Kingsley C, Lebel M, Pathak S, DePinho RA, Chang S. Elevated telomere-telomere recombination in WRN-deficient, telomere dysfunctional cells promotes escape from senescence and engagement of the ALT pathway. Genes Dev 2005; 19:2560-2570.

12. Wu L, Multani AS, He H, Cosme-Blanco W, Deng Y, Deng JM, Bachilo O, Pathak S, Tahara H, Bailey SM, Deng Y, Behringer RR,
Chang S. Pot1 deficiency initiates DNA damage checkpoint activation and aberrant homologous recombination at telomeres. Cell 2006; 126:49-62.

13. Celli GB, Denchi EL, de Lange T. Ku70 stimulates fusion of dysfunctional telomeres yet protects chromosome ends from homologous recombination. Nat Cell Biol 2006; 8:885-890.

14. Rai R, Zheng H, He H, Luo Y, Multani A, Carpenter PB, Chang $\mathrm{S}$. The function of classical and alternative non-homologous endjoining pathways in the fusion of dysfunctional telomeres. EMBO J 2010; 29:2598-2610.

15. Dragalla RC, Zhou J, Idate RR, Battaglia CLR, Liber HL, Bailey SM. Regulatory roles of tankyrase 1 at telomeres and in DNA repair: suppression of T-SCE and stabilization of DNA-PKcs. Aging 2010; 2 : this issue.

16. Smith S, Giriat I, Schmitt A, de Lange T. Tankyrase, a poly(ADP-ribose) polymerase at human telomeres. Science 1998; 282:1484-1487.

17. Smith $\mathrm{S}$, de Lange T. Tankyrase promotes telomere elongation in human cells. Curr Biol 2000; 10:1299-1302.

18. Denchi EL, de Lange T. Protection of telomeres through independent control of ATM and ATR by TRF2 and POT1. Nature 2007; 448:1068-1071.

19. Guo $X$, Deng $Y$, Lin $Y$, Cosme-Blanco $W$, Chan $S$, He H, Yuan $\mathrm{G}$, Brown EJ, Chang S. Dysfunctional telomeres activate an ATMATR-dependent DNA damage response to suppress tumorigenesis. EMBO J 2007; 26:4709-4719.

20. Deng $Y$, Guo X, Ferguson DO, Chang S. Multiple roles for MRE11 at uncapped telomeres. Nature 2009; 460:914-918.

21. Attwooll $\mathrm{CL}$, Akpinar $\mathrm{M}$, Petrini JH. The mre11 complex and the response to dysfunctional telomeres. Mol Cell Biol 2009; 29:5540-5551.

22. Dimitrova N, Chen YC, Spector DL, de Lange T. 53BP1 promotes non-homologous end joining of telomeres by increasing chromatin mobility. Nature 2008; 456:524-528.

23. Dimitrova N, de Lange T. Cell cycle-dependent role of MRN at dysfunctional telomeres: ATM signaling-dependent induction of nonhomologous end joining (NHEJ) in $\mathrm{G1}$ and resectionmediated inhibition of NHEJ in G2. Mol Cell Biol 2009; 29:55525563.

24. Haber JE. Alternative endings. Proc Natl Acad Sci U S A 2008; 105:405-406.

25. Bombarde O, Boby C, Gomez D, Frit P, Giraud-Panis MJ, Gilson E, Salles B, Calsou P. TRF2/RAP1 and DNA-PK mediate a double protection against joining at telomeric ends. EMBO J 2010; 29:1573-1584.

26. Hagelstrom RT, Blagoev KB, Niedernhofer $\sqcup$, Goodwin EH, Bailey SM. Hyper telomere recombination accelerates replicative senescence and may promote premature aging. Proc Natl Acad Sci U S A 2010; 107:15768-15773.

27. Chang S, Multani AS, Cabrera NG, Naylor ML, Laud $P$, Lombard D, Pathak S, Guarente L, DePinho RA. Essential role of limiting telomeres in the pathogenesis of Werner syndrome. Nat Genet 2004; 36:877-882.

28. Blagoev KB, Goodwin EH. Telomere exchange and asymmetric segregation of chromosomes can account for the unlimited proliferative potential of ALT cell populations. DNA Repair (Amst) 2008; 7:199-204.

29. Henson JD, Reddel RR. Assaying and investigating Alternative Lengthening of Telomeres activity in human cells and cancers. FEBS Lett 2010; 584:3800-3811. 
30. Cosme-Blanco W, Shen MF, Lazar AJ, Pathak S, Lozano G, Multani AS, Chang S. Telomere dysfunction suppresses spontaneous tumorigenesis in vivo by initiating p53-dependent cellular senescence. EMBO Rep 2007; 8:497-503.

31. Feldser DM, Greider CW. Short telomeres limit tumor progression in vivo by inducing senescence. Cancer Cell 2007; 11:461-469.

32. Fong PC, Boss DS, Yap TA, Tutt A, Wu P, Mergui-Roelvink $M$, Mortimer P, Swaisland H, Lau A, O'Connor MJ, Ashworth A, Carmichael J, Kaye SB, et al. Inhibition of poly(ADP-ribose) polymerase in tumors from BRCA mutation carriers. N Engl J Med 2009; 361:123-134.

33. McCabe N, Cerone MA, Ohishi T, Seimiya H, Lord CJ, Ashworth A. Targeting Tankyrase 1 as a therapeutic strategy for BRCA-associated cancer. Oncogene 2009; 28:1465-1470. 